\title{
Degree of Collaterals and Not Time Is the Determining Factor of Core Infarct Volume within 6 Hours of Stroke Onset
}

\author{
E. Cheng-Ching, J.A. Frontera, S. Man, J. Aoki, Y. Tateishi, F.K. Hui, D. Wisco, P. Ruggieri, M.S. Hussain, and K. Uchino
}

\begin{abstract}
BACKGROUND AND PURPOSE: Growth of the core infarct during the first hours of ischemia onset is not well-understood. We hypothesized that factors other than time from onset of ischemia contribute to core infarct volume as measured by MR imaging.
\end{abstract}

MATERIALS AND METHODS: Prospectively collected clinical and imaging data of consecutive patients with stroke presenting between March 2008 and April 2013 with anterior circulation large-vessel occlusion and MR imaging performed within 6 hours from the time of onset were reviewed. The association of time from onset, clinical, and radiographic features with DWI volume was assessed by using $\chi^{2}$ and Mann-Whitney $U$ tests.

RESULTS: Of 91 patients, 21 (23\%) underwent MR imaging within 0-3 hours from onset, and 70 (76\%), within 3-6 hours. Median MR imaging infarct volume was similar in both timeframes, (24.7 versus $29.4 \mathrm{~mL}, P=.906$ ), and there was no difference in the proportion of patients with large infarct volumes ( $\geq 70 \mathrm{~mL}, 23.8 \%$ versus $22.8 \%, P=.928$ ). Using receiver operating characteristic analysis, we detected no association between the time from onset and MR imaging infarct volume (area under the curve $=0.509$ ). In multivariate analysis, CTA collaterals ( $>50 \%$ of the territory) (adjusted OR, 0.192; 95\% Cl, 0.04-0.9; $P=.046$ ), CTA ASPECTS (adjusted OR, 0.464; 95\% Cl, 0.3-0.8; $P=$ .003 ), and a history of hyperlipidemia (adjusted OR, 11.0; $95 \% \mathrm{Cl}, 1.4-88.0 ; P=.023$ ) (but not time from stroke onset to imaging) were independent predictors of MR imaging infarct volume.

CONCLUSIONS: Collateral status but not time from stroke onset to imaging was a predictor of the size of core infarct in patients with anterior circulation large-vessel occlusion presenting within 6 hours from onset.

ABBREVIATIONS: IQR = interquartile range; $L S W=$ last seen well

ntravenous tPA administered within 4.5 hours from symptom onset improves clinical outcomes in patients with acute ischemic stroke. ${ }^{1,2}$ The importance of time from stroke onset to thrombolysis has been extensively demonstrated as a predictor of improved outcomes. The National Institute of Neurologic Disorders and Stroke tPA trial and pooled analysis from major randomized stroke IV tPA trials showed that late tPA administration is associated with lower likelihood of good clinical outcomes. ${ }^{3}$ In a cohort of patients treated with intravenous/intra-arterial therapy,

Received October 12, 2014; accepted after revision January 6, 2015.

From the Cerebrovascular Center (E.C.-C., J.A.F., S.M., F.K.H., D.W., M.S.H., K.U.) and Department of Neuroradiology (P.R.), Imaging Institute, Cleveland Clinic, Cleveland, Ohio; Department of Neurology and Cerebrovascular Disease (J.A.), Nippon Medical School, Tokyo, Japan; and Cerebrovascular Center (Y.T.), Department of Neurology and Strokology, Nagasaki University Hospital, Nagasaki, Japan.

Please address correspondence to Ken Uchino, MD, Cerebrovascular Center, Neurological Institute, Cleveland Clinic, 9500 Euclid Ave, S-80, Cleveland, OH 44195;

e-mail: uchinok@ccf.org

三 Indicates article with supplemental on-line table.

http://dx.doi.org/10.3174/ajnr.A4274
Khatri et $\mathrm{al}^{4}$ demonstrated that the probability of good clinical outcome following angiographic reperfusion is time-dependent, with a reduced probability of good outcome as the time to recanalization increases. Despite the importance of time from clinical stroke onset to treatment, other parameters play a major role in influencing clinical outcome, including age and baseline NIHSS score. Infarct volume at baseline has also been demonstrated to be a strong predictor of clinical outcome..$^{5-8}$ The association between time from stroke onset to presentation and core infarct volume is unclear, and factors other than time may have a major influence in the first hours from ischemia onset. A recently published study analyzed a group of patients with anterior circulation large-vesselocclusion strokes who underwent MR imaging with DWI within 30 hours from stroke onset and found no correlation between the time of onset and stroke volume. The authors suggested that other factors more powerful than time (such as collateral circulation) determine infarct size in these first 30 hours. ${ }^{9}$ It is important to determine what relationship exists between time and infarct volume, especially in the early hours from stroke onset because this 


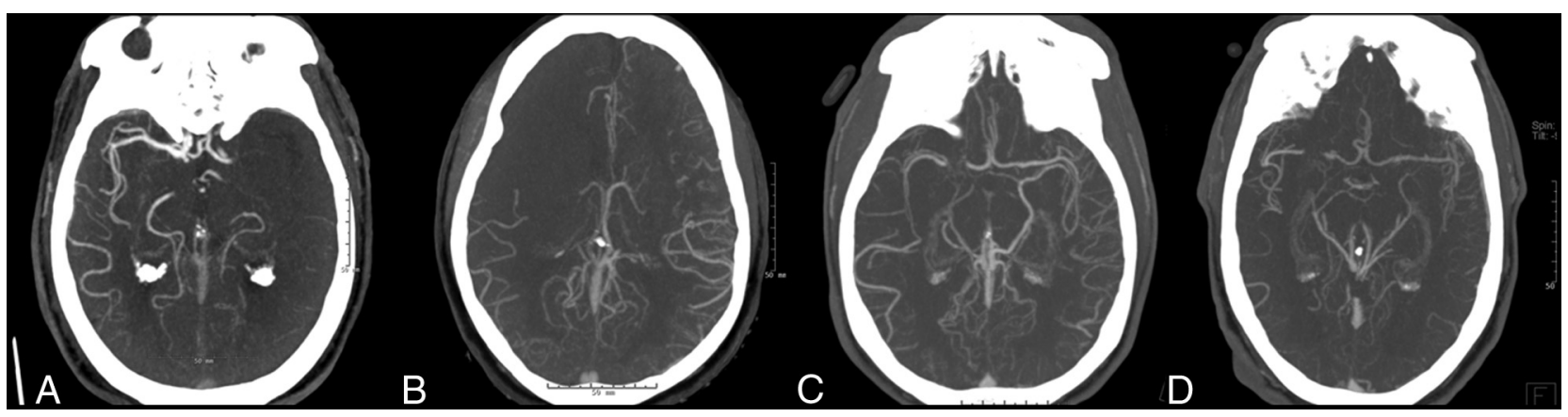

FIG 1. CTA collateral status in the region of the occluded vessel. A, Absent collaterals. B, Minimal ( $<50 \%)$ collaterals. C, Partial ( $>50 \%)$ collaterals. $D$, Full presence of collaterals.

may have implications in the decisions for imaging selection and treatment. The goal of this study was to explore the relationship of DWI lesion volume to time from stroke onset to imaging within the first 6 hours and to describe imaging characteristics in anterior circulation large-vessel-occlusion strokes at 0-6 hours from the time the patients were last seen well (LSW). We further assessed potential variables that may influence core infarct volume other than time and sought to evaluate predictors of small and large infarct volumes independent of the time factor.

\section{MATERIALS AND METHODS \\ Study Population}

Using a prospectively collected acute stroke data base, we retrospectively identified consecutive patients with acute ischemic stroke admitted to our institution between March 2008 and April 2013. We included only patients with anterior circulation strokes and large-artery occlusion on CTA (involving the intracranial ICA and the M1 or M2 segment of the MCA) in whom MR imaging of the brain was performed within 6 hours from the time LSW and before any endovascular reperfusion therapy was initiated. A reported witnessed time of LSW was required for inclusion, and this time point was used as a surrogate for the time of stroke onset. Baseline clinical characteristics were systematically collected, including demographic data, stroke risk factors, and admission NIHSS scores.

\section{Acute Imaging}

At our institution, in every patient who presents with an acute ischemic stroke within 8 hours from symptom onset in whom the NIHSS score is $\geq 8$, we perform a noncontrast CT and CTA if no contraindications exist. If a large-vessel occlusion (ICA, the M1 and/or M2 segment of the MCA, basilar occlusion) is detected and there are no contraindications, a "hyperacute" MR imaging of the brain is performed immediately to assess infarct volume and to evaluate the region of ischemic penumbra.

Noncontrast CT is performed with a multisection CT scanner (Somatom Sensation 64/16; Siemens, Erlangen, Germany) with 1.2-mm collimation, 4.5-mm sections (Sensation 64); and 1.5 -mm collimation, $4.8-\mathrm{mm}$ sections (Sensation 16). CTA is performed with a helical scan technique, with acquisitions obtained after a single intravenous bolus injection of $80 \mathrm{~mL}$ of nonionic contrast (iopromide, Ultravist 370; Bayer Healthcare LLC, Whippany, New Jersey) into an antecubital vein at a rate of $4 \mathrm{~mL} / \mathrm{s}$. The CTA acquisition includes the region of the aortic arch to the centrum semiovale or the vertex with spiral $0.6-\mathrm{mm}$ collimation, 0.6-mm section thickness, 0.6-mm interval (Sensation 64); and spiral $0.75-\mathrm{mm}$ collimation, $0.75-\mathrm{mm}$ section thickness, and 0.75 -mm interval (Sensation 16). MR imaging studies were performed by using a commercially available echo-planar instrument on a 1.5T Magnetom Aera system with software version, syngo MR D11 (Siemens). The neuroimaging protocol for acute stroke in our hospital includes a diffusion-weighted imaging sequence (TR/TE, 3500/114 ms; b-values, 0 and $1400 \mathrm{~s} / \mathrm{mm}^{2}$; FOV, 230 $\mathrm{mm}$; section thickness, $5.0 \mathrm{~mm}$; base resolution, 128; bandwidth, $1260 \mathrm{~Hz}$ ); a FLAIR sequence (TR/TE, 8360/94 ms; TI, 2200 ms; flip angle, $180^{\circ}$; FOV, $210 \mathrm{~mm}$; section thickness, $4.0 \mathrm{~mm}$; base resolution, 256; bandwidth, $208 \mathrm{~Hz}$ ); and a dynamic susceptibility contrast PWI sequence (TR/TE, 2000/45 ms; flip angle, $90^{\circ}$; FOV, $230 \mathrm{~mm}$; section thickness, $5.0 \mathrm{~mm}$; base resolution, 128; bandwidth, $1260 \mathrm{~Hz}$ ).

\section{Imaging Assessment}

An experienced neuroradiologist (F.K.H.) led and oversaw the imaging analysis. Admission pretreatment noncontrast CT and CTA were reviewed, and an Alberta Stroke Program Early CT Score was calculated. Noncontrast CT images were not available for ASPECTS evaluation in 10 patients ( 1 from 0 to $<3$ hours, and 9 from 3 to $<6$ hours). Pretreatment CTA collateral status was analyzed and classified into absent, minimal $(<50 \%)$, partial $(>50 \%)$, and full presence of collateral branches opacifying the major intracranial vascular territory that normally supplies the region of the acute infarct (Fig 1). ${ }^{10}$ CTA was not available for collateral vessel evaluation in 15 patients ( 2 from 0 to $<3$ hours, and 13 from 3 to $<6$ hours). Under the direct supervision of a neuroradiologist (F.K.H.), 4 vascular neurologists (J.A., Y.T., E.C.-C., and S.M.), blinded to the clinical information, reviewed and analyzed the MR images by using commercial software (syngoMMWP VE36A, syngoVE32D, WinNT 5.2, Service Pack 2; Siemens) on a dedicated workstation. Infarct volumes were obtained from DWI. The area of the infarct on each section was obtained by the software after manually outlining the perimeter of the lesion. The infarct volume was calculated by adding the infarct areas on each section and multiplying by the section thickness.

\section{Statistical Analysis}

The cohort of patients was divided into groups according to time intervals from LSW to MR imaging ( $0-3$ hours versus 3-6 hours). We used a cutoff of $70 \mathrm{~mL}$ of DWI infarct volume to group pa- 


\begin{tabular}{|c|c|c|c|c|}
\hline & $\begin{array}{c}0 \text { to }<3 \text { Hours } \\
(n=21)\end{array}$ & $\begin{array}{c}\text { 3-6 Hours } \\
(n=70)\end{array}$ & $\begin{array}{c}\text { 0-6 Hours } \\
\text { (Entire Cohort) } \\
(n=91)\end{array}$ & $\begin{array}{c}P \text { Value } \\
\text { (Difference, } \\
0-3 \text { vs } 3-6)\end{array}$ \\
\hline Age (yr) (median) (IQR) & $58(54-77)$ & $73(63.25-83)$ & $70(59-82)$ & .034 \\
\hline NIHSS (median) (IQR) & $13(11-19)$ & $17(13-22.75)$ & $17(12-21.5)$ & .050 \\
\hline CT ASPECTS (median) (IQR) & $9(7-10)$ & $8(16-9)$ & $8(6-9)$ & .202 \\
\hline CT ASPECTS $\geq 7$ (No.) (\%) & $17 / 20(85 \%)$ & $42 / 61(69 \%)$ & $59 / 81(72 \%)$ & .159 \\
\hline CTA collaterals $>50 \%$ (No.) (\%) & $14 / 19(74 \%)$ & $37 / 57(65 \%)$ & $51 / 76(67 \%)$ & .481 \\
\hline DWI volume (mL) (median) (IQR) & $24.7(13.9-63.8)$ & $29.4(12.1-68.5)$ & $29.2(12.3-68.5)$ & .906 \\
\hline MRI DWI volume $\geq 70 \mathrm{~mL}$ of DWI volume (No.) (\%) & $5(23.8 \%)$ & $16(22.8 \%)$ & $21(22.8 \%)$ & .928 \\
\hline MRI DWI volume $\geq 100 \mathrm{~mL}$ of DWI volume (No.) (\%) & $4(19 \%)$ & $13(18.6 \%)$ & $17(18.7 \%)$ & .961 \\
\hline Terminal ICA occlusion (No.) (\%) & $5(23.8 \%)$ & $18(25.7 \%)$ & $23(25.3 \%)$ & .961 \\
\hline Proximal Ml occlusion (No.) (\%) & $5(23.8 \%)$ & $21(30 \%)$ & $26(28.6 \%)$ & .706 \\
\hline Distal M1 occlusion (No.) (\%) & $11(52.4 \%)$ & $31(44.3 \%)$ & $42(46.1 \%)$ & .393 \\
\hline
\end{tabular}

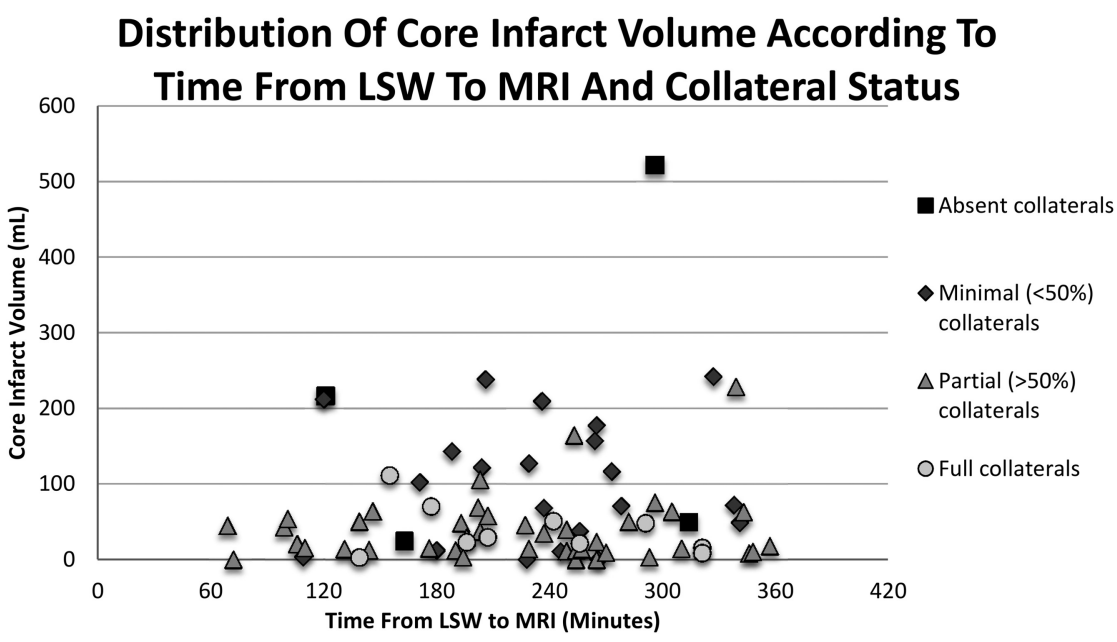

FIG 2. Distribution of DWI infarct volume of each patient according to the time from last seen well to MR imaging and collateral status.

tients into large infarcts $(\geq 70 \mathrm{~mL})$ and small infarcts $(<70 \mathrm{~mL})$, and these groups were compared. ${ }^{8}$

Demographics, medical history, and clinical and radiographic features were compared between patients who underwent early MR imaging ( $0-3$ hours from LSW) versus late MR imaging (3-6 hours from LSW) and between patients with large $(\geq 70 \mathrm{~mL})$ versus small $(<70 \mathrm{~mL})$ infarcts by using the Pearson $\chi^{2}$ and MannWhitney $U$ tests. A receiver operating characteristic curve was constructed to assess the sensitivity and specificity of predicting DWI volume on the basis of time from LSW to MR imaging (0-3 versus 3-6 hours). Backward stepwise multivariable logistic regression analysis was performed to identify independent predictors of DWI volume ( $\geq 70 \mathrm{~mL})$.

\section{RESULTS}

Ninety-one patients met the inclusion criteria and were included in our study analysis. The median age of the overall group was 70 years (interquartile range [IQR], 59-82 years), the median NIHSS score was 17 (IQR, 12-21.5), and the median CT ASPECTS was 8 (IQR, 6-9). The median time from LSW to MR imaging was 239 minutes (range, 69-357 minutes; IQR, 189-280 minutes). The mean DWI infarct volume for the entire group of patients was $57.9 \pm 77.9 \mathrm{~mL}$, with a median of $29.2 \mathrm{~mL}$ (IQR, 12.3-68.5 mL). The Table shows clinical, demographic, and radiographic characteristics of patients according to the time inter- val from LSW to MR imaging. When we compared patients presenting early who underwent MR imaging within $0-3$ hours from LSW and those patients who presented later who underwent MR imaging within 3-6 hours from LSW, the earlier group tended to be younger (median age, 58 years; IQR, $54-77$ years versus 73 years; IQR, 63.25-83 years; $P=$ $.034)$ and had lower NIHSS scores (median, 13; IQR, 11-19 versus 17; IQR, $13-$ 22.75; $P=.050)$. However, the median infarct volume was similar between early and late imaging groups $(24.7 \mathrm{~mL}$; IQR, 13.9-63.8 mL; and $29.4 \mathrm{~mL}$; IQR, $12.1-$ $68.5 \mathrm{~mL} ; P=.906)$. A large infarct $(\geq 70$ $\mathrm{mL}$ ) was seen in $23.8 \%$ of patients with imaging within 3 hours from LSW compared with $22.8 \%$ of patients who presented 3-6 hours from LSW $(P=.928)$. Similarly, there was no difference in rates of large infarcts by using a cut-point of $100 \mathrm{~mL}$ between those who underwent MR imaging within $0-3$ versus 3-6 hours (Table).

Eight patients presented very early, in whom an MR imaging was obtained $<2$ hours from LSW. In this very early group, the median DWI infarct volume was $18 \mathrm{~mL}$ (IQR, 5.6-43 mL), and none was larger than $70 \mathrm{~mL}$. From 2 to $<3$ hours from LSW, the median DWI infarct volume was $50.3 \mathrm{~mL}$ (IQR, 14.8-102.5 mL), and $38.4 \%$ had large infarcts ( $\geq 70 \mathrm{~mL}$ ). Figure 2 shows the DWI volume by time intervals from LSW to MR imaging and the degree of collaterals for each patient. A receiver operating characteristic curve assessing the time from LSW to MR imaging (0-3 versus 3-6 hours) and DWI volume was obtained with an area under the curve of 0.509 , demonstrating no association between time from LSW to MR imaging and DWI infarct volume (Fig 3).

The On-line Table shows demographic, clinical, and radiographic patient characteristics according to DWI infarct volume. Those with larger infarcts were more likely to have a history of hyperlipidemia ( $81 \%$ versus $40 \%, P=.049)$, coronary artery disease $(57 \%$ versus $11 \%, P=.027)$, and lower noncontrast CT ASPECTS (5.5 versus 9, $P<.001$ ) and CTA ASPECTS (5 versus 9, $P<.001)$. Patients with smaller infarcts $(\leq 70 \mathrm{~mL})$ were more likely to have at least partial ( $>50 \%$ of the territory) or better collaterals on CTA compared with those with large infarcts $(64 \%$ 


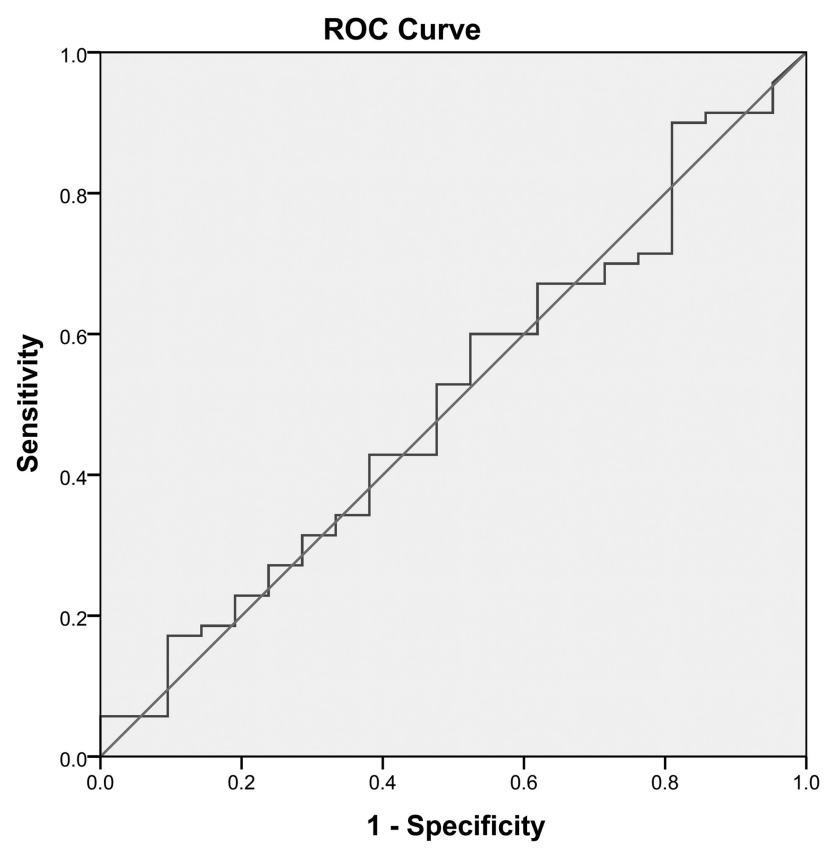

FIG 3. Receiver operating characteristic curve demonstrating no association between the time from LSW to MR imaging $(0-3$ hours versus 3-6 hours) and DWI infarct volume.

versus $29 \%, P=.001)$. The group with large DWI infarct volumes was more likely to have occlusion of the cervical ICA (29\% versus $6 \%$, $P=.018$ ), proximal M1 MCA segment occlusion (52\% versus $23 \%$, $P=.001)$, or either terminal ICA or proximal M1 occlusion $(57 \%$ versus $31 \%, P=.003$ ). The use of IV tPA before MR imaging and stroke mechanism were not associated with infarct volume.

In multivariate analysis, the time from stroke onset was not a predictor of infarct volume. CTA collaterals of $>50 \%$ of the territory involved (adjusted OR, 0.192; 95\% CI, 0.04-0.9; $P=.046$ ) and the CTA ASPECTS (adjusted OR, 0.464; 95\% CI, 0.3-0.8; $P=$ $.003)$ were negative independent predictors of large infarct volume ( $\geq 70 \mathrm{~mL}$ ). History of hyperlipidemia (adjusted OR, 11.0; 95\% CI, 1.4-88.0; $P=.023$ ) was an independent predictor of large infarcts. There was neither a significant interaction between a history of hyperlipidemia and CTA collaterals nor an association of baseline statin use with infarct volume.

\section{DISCUSSION}

In a cohort of patients with anterior circulation large-vessel occlusion imaged with DWI within 6 hours from stroke onset, we demonstrate that time to imaging is not a determinant of core infarct as measured by DWI. Hakimelahi et $\mathrm{al}^{9}$ found no correlation between infarct volume and time after stroke onset within 30 hours from presentation. We confirmed this finding in a group of patients imaged at an earlier timeframe from stroke onset that is more clinically relevant for decision-making in regard to endovascular intervention. We found that large infarct volumes can be seen in patients presenting equally early and late within the time studied, and we were able to verify that collateral status does indeed have a significant impact on infarct volume. The clinical implications of our study include the possibilities of both safer patient selection for endovascular reperfusion within conventional time windows and extension of these treatment windows.
The definition of large stroke was based on a DWI lesion volume of at least $70 \mathrm{~mL}$. We were also unable to detect a difference in DWI volume by using a larger threshold of $100 \mathrm{~mL}$ between those who underwent MR imaging between $0-3$ versus 3-6 hours. These 2 values have been demonstrated to be significant thresholds impacting clinical outcome in patients with stroke with anterior circulation large-vessel occlusions. ${ }^{8,11,12}$ A study by using MR imaging-based selection of patients with anterior circulation large-vessel-occlusion ischemic stroke demonstrated that the best clinical outcomes and least infarct growth occur in those with DWI infarct volume of $<70 \mathrm{~mL}$ who underwent early recanalization and that an infarct of $70 \mathrm{~mL}$ is the threshold above which patients do poorly despite treatment. ${ }^{8}$ The Diffusion and Perfusion Imaging Evaluation for Understanding Stroke Evolution trial demonstrated that MR imaging selection can identify patients who may benefit from reperfusion therapy (such as those with target mismatch and likely smaller DWI infarct volumes), and at the same time, it showed that patients with a large DWI lesion (malignant profile with DWI lesion volume $>100 \mathrm{~mL}$ and/or PWI of $>100 \mathrm{~mL}$ with time-to-maximum delay of $>8$ seconds) had a high risk of symptomatic intracerebral hemorrhage with recanalization, suggesting that patients with large strokes should not receive reperfusion therapy. ${ }^{11,12}$ Identification of large DWI infarcts in a similar proportion of patients presenting at early and late timeframes suggests the potential importance of imaging selection for reperfusion therapy among patients presenting within 6 hours from stroke onset. The impact of DWI infarct volume on clinical outcomes with and without reperfusion therapy has been previously described..$^{5-8,11}$ Wisco et $\mathrm{al}^{13}$ demonstrated that MR imaging patient selection may reduce the number of futile and harmful interventions; this reduction may translate to better clinical outcomes for patients without revascularization. In our study, nearly $25 \%$ of patients who underwent MR imaging within 3 hours of stroke onset had large acute strokes with volumes of $>70 \mathrm{~mL}$. These data imply that early reperfusion therapy in all patients without imaging selection may yield a substantial proportion of patients who will not do well, and this implication should be taken into account in clinical practice and in the design of future clinical trials.

The findings of this study should not undermine the importance of time to treatment as a major factor influencing clinical outcomes and the importance of shorter time to treatment, which has been extensively demonstrated. ${ }^{1,2,4,14}$ We observed that those presenting ultra-early, within 2 hours from onset, had core infarct volumes of $<70 \mathrm{~mL}$. It can be argued that this ultra-early patient population with small infarct volumes can potentially benefit the most from reperfusion therapies, suggesting that very early reperfusion therapy may have the greatest impact on outcomes in anterior circulation largevessel-occlusion ischemic strokes. In this very early group, we did not find large infarcts, but the number of patients was small.

When we compared large and small strokes, the proportion of patients presenting within $0-3$ hours from onset was not significantly different between the 2 groups, while the degree of collateral circulation and a history of hyperlipidemia were found to be independent predictors of large infarct volumes. In a retrospective study, Menon et $\mathrm{al}^{15}$ showed that better CTA collateral status was associated with higher follow-up CT ASPECTS and lower 3-month modified Rankin Scale scores. In a study with a small 
number of patients presenting with anterior circulation large-vessel-occlusion ischemic strokes who underwent CTA evaluation and intra-arterial therapy, Angermaier et $\mathrm{al}^{16}$ demonstrated that reperfusion and the extent of CTA collaterals (but not time to treatment) were independent predictors of final infarct volume on follow-up noncontrast CT. In the setting of an arterial occlusion, collateral circulation may provide alternative routes of blood supply to the parenchyma in the distribution of the occluded vessel and potentially limit the size of the infarcted core and penumbra, at least temporarily. During the first minutes to hours after an acute arterial occlusion, collateral circulation may be the only source of perfusion; therefore, the presence of poor collateral status on admission may be associated with large strokes independent of the time elapsed from the onset of the occlusion. In a study of patients with anterior circulation large-artery-occlusion ischemic strokes presenting within 9 hours from onset of symptoms, Souza et $\mathrm{al}^{17}$ showed that a malignant CTA collateral profile (absent collaterals) strongly correlated and was highly specific for large DWI lesions on admission MR imaging.

We found the large-infarct group to be associated with a history of hyperlipidemia. This finding may be a type 1 error from multiple comparisons because it cannot be explained and has not been reported previously, to our knowledge. Menon et $\mathrm{al}^{18}$ reported an association between metabolic syndrome and poor leptomeningeal collateral status in a group of patients presenting with acute ischemic stroke and large anterior circulation vessel occlusion in South Korea; however, no causative explanation was studied, and further investigation was recommended. It is possible that hyperlipidemia may be a maker of vasculopathy and dysfunctional autoregulation with a reduced capacity of collateral recruitment; however, this possibility cannot be assessed in this retrospective study and requires further investigation.

This study has several limitations. It has the inherent limitations of a retrospective observational study. The sample size was small, especially when breaking down the cohort into groups by time intervals, in which we see a smaller number of patients presenting in the early times. An exact time of symptom onset was not available in all patients; therefore, the time from LSW was used as the most accurate surrogate for time of stroke-symptom onset. Even though using the time of LSW may overestimate the actual time interval between onset and imaging, an overestimation is preferred rather than an underestimation, given the focus of our study in detecting large strokes at early time points. Using the time of LSW to MR imaging from 0 to 6 hours, we are certain that stroke onset occurred within this timeframe; therefore, our cohort consists of patients with a well-established time of stroke onset to MR imaging within the time window criteria for this study. This patient group correlates with real-world clinical care in which the exact time of onset is frequently unknown, but the time of LSW is used more often when deciding treatment for these patients.

\section{CONCLUSIONS}

In this cohort, time from stroke onset to imaging was not a determinant of core infarct size in patients with stroke with anterior circulation large-vessel occlusion within the first 6 hours. Collateral status and the CTA ASPECTS are independent predictors of infarct volume within this period.
Disclosures: Esteban Cheng-Ching-UNRELATED: Royalties: Lippincott Williams \& Wilkins, Comments: royalties for authorship of the book Comprehensive Review in Clinical Neurology.

\section{REFERENCES}

1. The National Institute of Neurological Disorders and Stroke rt-PA Stroke Study Group. Tissue plasminogen activator for acute ischemic stroke. $N$ Engl J Med 1995;333:1581-87

2. Hacke W, Kaste M, Bluhmki E, et al. Thrombolysis with alteplase 3 to 4.5 hours after acute ischemic stroke. $N$ Engl J Med 2008; 359:1317-29

3. Lees KR, Bluhmki E, von Kummer R, et al. Time to treatment with intravenous alteplase and outcome in stroke: an updated pooled analysis of ECASS, ATLANTIS, NINDS, and EPITHET trials. Lancet 2010;375:1695-703

4. Khatri P, Abruzzo T, Yeatts SD, et al. Good clinical outcome after ischemic stroke with successful revascularization is time-dependent. Neurology 2009;73:1066-72

5. Sanák D, Nosál' V, Horák D, et al. Impact of diffusion-weighted MRI-measured initial cerebral infarction volume on clinical outcome in acute stroke patients with middle cerebral artery occlusion treated by thrombolysis. Neuroradiology 2006;48:632-39

6. Thijs VN, Lansberg MG, Beaulieu C, et al. Is early ischemic lesion volume on diffusion-weighted imaging an independent predictor of stroke outcome? A multivariable analysis. Stroke 2000;31:2597-602

7. Vogt G, Laage R, Shuaib A, et al. Initial lesion volume is an independent predictor of clinical stroke outcome at day 90: an analysis of the Virtual International Stroke Trials Archive (VISTA) database. Stroke 2012;43:1266-72

8. Yoo AJ, Verduzco LA, Schaefer PW, et al. MRI-based selection for intra-arterial stroke therapy: value of pretreatment diffusionweighted imaging lesion volume in selecting patients with acute stroke who will benefit from early recanalization. Stroke 2009; 40:2046-54

9. Hakimelahi R, Vachha BA, Copen WA, et al. Time and diffusion lesion size in major anterior circulation ischemic strokes. Stroke 2014;45:2936-41

10. Tan JC, Dillon WP, Liu S, et al. Systematic comparison of perfusion-CT and CT-angiography in acute stroke patients. Ann Neurol 2007;61:533-43

11. Albers GW, Thijs VN, Wechsler L, et al. Magnetic resonance imaging profiles predict clinical response to early reperfusion: the Diffusion and Perfusion Imaging Evaluation for Understanding Stroke Evolution (DEFUSE) study. Ann Neurol 2006;60:508-17

12. Lansberg MG, Straka M, Kemp S, et al. MRI profile and response to endovascular reperfusion after stroke (DEFUSE 2): a prospective cohort study. Lancet Neurol 2012;11:860-67

13. Wisco D, Uchino K, Saqqur M, et al. Addition of hyperacute MRI AIDS in patient selection, decreasing the use of endovascular stroke therapy. Stroke 2014;45:467-72

14. Hacke W, Donnan G, Fieschi C, et al. Association of outcome with early stroke treatment: pooled analysis of ATLANTIS, ECASS, and NINDS rt-PA stroke trials. Lancet 2004;363:768-74

15. Menon BK, Smith EE, Modi J, et al. Regional leptomeningeal score on CT angiography predicts clinical and imaging outcomes in patients with acute anterior circulation occlusions. AJNR Am J Neuroradiol 2011;32:1640-45

16. Angermaier A, Langner S, Kirsch M, et al. CT-angiographic collateralization predicts final infarct volume after intra-arterial thrombolysis for acute anterior circulation ischemic stroke. Cerebrovasc Dis 2011;31:177-84

17. Souza LC, Yoo AJ, Chaudhry ZA, et al. Malignant CTA collateral profile is highly specific for large admission DWI infarct core and poor outcome in acute stroke. AJNR Am J Neuroradiol 2012;33:1331-36

18. Menon BK, Smith EE, Coutts SB, et al. Leptomeningeal collaterals are associated with modifiable metabolic risk factors. Ann Neurol 2013;74:241-48 